\title{
Composição centesimal e valor calórico de peixes Clupeiformes na costa norte do Rio de Janeiro
}

\author{
Danielle Rodrigues Awabdi ${ }^{1}$ \\ Marcello Pimentel Fonseca de Carvalho ${ }^{1}$ \\ Vanessa Trindade Bittar ${ }^{1}$ \\ Manuel Vazquez Vidal Júnior ${ }^{2}$ \\ Ana Paula Madeira Di Beneditto ${ }^{1 *}$ \\ Universidade Estadual do Norte Fluminense \\ ${ }^{1} \mathrm{CBB}$, Laboratório de Ciências Ambientais \\ CEP 28013-620, Campos dos Goytacazes - RJ, Brasil \\ ${ }^{2}$ CCTA, Laboratório de Zootecnia e Nutrição Animal \\ *Autor para correspondência \\ anapaula@uenf.br
}

\section{Resumo}

O objetivo deste trabalho é fornecer informações sobre a composição centesimal e o valor calórico dos peixes Clupeiformes Chirocentrodon bleekerianus, Pellona harroweri e Lycengraulis grossidens. Os espécimes foram coletados entre 2007 e 2010 na costa norte do Estado do Rio de Janeiro. Análises bromatológicas (proteína, lipídio e matéria mineral) foram realizadas em amostras compostas; o teor de carboidrato e a energia bruta foram calculados a partir de relações entre as variáveis. As espécies de peixes foram significativamente distintas em relação ao porte (comprimento total e peso), composição centesimal e energia bruta; apenas o teor de carboidrato foi similar. A espécie L. grossidens foi representada por indivíduos maiores e com teor de lipídio superior, enquanto $C$. bleekerianus e $P$. harroweri apresentaram os maiores percentuais de proteína e matéria mineral, além de valores de energia bruta mais elevados. O percentual de proteína foi diretamente proporcional ao valor de energia bruta, enquanto o percentual de lipídio apresentou relação inversa com essas duas variáveis. Este estudo servirá de base para o entendimento da seletividade alimentar de carnívoros marinhos em regiões costeiras e para a elaboração de modelos de bioenergética.

Palavras-chave: Clupeiformes, Composição centesimal, Energia bruta, Oceano Atlântico Ocidental

\section{Abstract}

Proximate composition and caloric value of Clupeiformes fishes from the Northern Rio de Janeiro coast. The aim of this study is to provide information on the proximate composition and caloric value of the Clupeiformes fishes Chirocentrodon bleekerianus, Pellona harroweri, and Lycengraulis grossidens. The specimens were collected between 2007 and 2010 in Northern Rio de Janeiro. The bromatological analysis (protein, lipid, and mineral matter) was performed on composite samples; carbohydrate content and gross energy were calculated from relationships between variables. The fish species were significantly different in size (total length and weight), proximate composition, and gross energy; only the carbohydrate content was similar. The species $L$. grossidens was represented by larger individuals with higher lipid content, as C. bleekerianus and $P$. 
harroweri had the highest percentages of protein and mineral matter, as well as the highest gross energy values. The percentage of protein was directly proportional to the amount of gross energy, as the percentage of lipid showed an inverse relationship with these two variables. This study provides a basis for understanding the food selectivity of marine carnivores in coastal regions and elaborating bioenergetics models.

Key words: Clupeiformes, Gross energy, Proximate composition, Western Atlantic Ocean

\section{Introdução}

A ordem Clupeiformes reúne mais de 400 espécies de teleósteos pelágicos de pequeno porte que se distribuem em todo o mundo (FROESE; PAULY, 2010). A maior parte das espécies habita águas marinhas costeiras, com vários grupos eurialinos e anádromos, mas há espécies se distribuindo permanentemente em corpos de água doce (FIGUEIREDO; MENEZES, 1978; WHITEHEAD, 1985). A alimentação desses peixes inclui uma variedade de organismos fito- e zooplanctônicos e juvenis de peixes e crustáceos (FROESE; PAULY, 2010). Os Clupeiformes contribuem com cerca de $25 \%$ do total anual de peixes capturados comercialmente no mundo (FAO, 2010) e, além da participação na economia mundial, também representam importantes componentes de cadeias tróficas aquáticas, fazendo parte da dieta de peixes (DENNERLINE; VAN DEN AVYLE, 2000; CYTERSKI et al., 2002; CAMPO et al., 2006), aves (DÄNHARDT; BECKER, 2011) e mamíferos (NILSSEN, 1995; BEARZIA et al., 2006).

A análise da contribuição nutricional e calórica das presas em relação aos requerimentos dos predadores é uma das ferramentas que pode ser utilizada na interpretação da dieta e dos padrões de seleção alimentar (EGGLETON; SCHRAMM JR., 2004; DOYLE et al., 2007; DI BENEDITTO et al., 2009). Modelos de bioenergética permitem predições sobre o comportamento e a dinâmica populacional de muitos predadores (ACEVEDO-GUTIERREZ et al., 2002; BURKE; RICE, 2002), e são particularmente úteis ao estudo de populações cuja observação direta em seu hábitat é difícil, como no caso de animais aquáticos.

Nesse contexto, o presente trabalho contribui com informações sobre a composição centesimal e energética de três espécies de Clupeiformes (Chirocentrodon bleekerianus Poey, 1867, Pellona harroweri Fowler, 1917 e Lycengraulis grossidens Spix \& Agassiz, 1829) comuns em regiões costeiras do oceano Atlântico Ocidental (FROESE; PAULY, 2010), onde são registradas como presas preferenciais de carnívoros marinhos (DI BENEDITTO; RAMOS, 2001; BITTAR et al., 2008).

\section{Material e Métodos}

Os peixes foram coletados entre 2007 e 2010, em amostragens anuais realizadas no período de primaveraverão $(\mathrm{n}=2)$ e outono-inverno $(\mathrm{n}=2)$, a partir da pesca artesanal com rede de parelha conduzida nas proximidades da foz do Rio Paraíba do Sul, costa norte do Rio de Janeiro. Esse artefato opera do fundo a meiaágua e a prática pesqueira envolve duas embarcações que se deslocam simultaneamente e lado-a-lado, arrastando a rede através do campo de pesca. $\mathrm{O}$ artefato possui formato cônico com 24-30m de comprimento, $20 \mathrm{~m}$ de abertura na boca e $6 \mathrm{~cm}$ de malha. Os arrastos para a coleta dos peixes estudados foram realizados paralelamente à linha de costa, entre $21^{\circ} 30^{\prime} \mathrm{S}$ e $21^{\circ} 40^{\prime} \mathrm{S}$, a partir de menos de um (1) até $5 \mathrm{~km}$ de distância da costa, em profundidades que variaram de 10 a $15 \mathrm{~m}$ (Figura 1). Os Clupeiformes analisados não possuem valor comercial na região e são considerados como capturas acidentais desse tipo de pescaria, que tem como principais alvos espécies da família Sciaenidae.

Todos os espécimes de C. bleekerianus, P. harroweri e L. grossidens capturados foram acondicionados em caixa de isopor com gelo e transferidos ao laboratório logo após a pescaria. Após cada coleta, os peixes foram medidos em relação ao comprimento total $(0,1 \mathrm{~mm})$ e peso $(0,1 \mathrm{~g})$. Espécimes com porte similar foram agrupados para a formação de amostras compostas, de modo a se obter massa suficiente para a realização das análises bromatológicas (proteína, lipídio e matéria mineral). Cada amostra composta foi formada por três a seis indivíduos que, após a coleta, ainda frescos, eram 


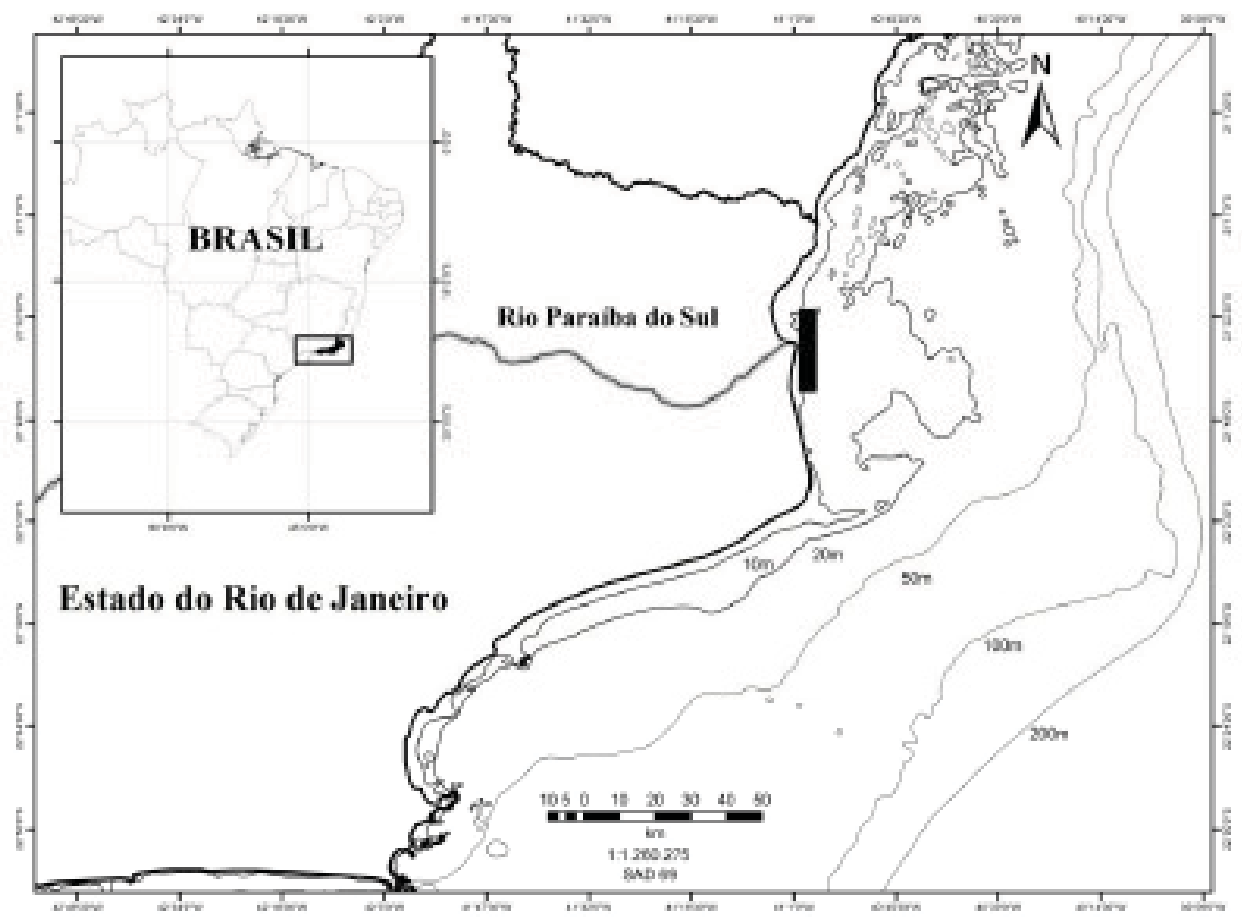

FIGURA 1: Costa norte do Rio de Janeiro, com indicação do Rio Paraíba do Sul e do perfil batimétrico. A área de coleta dos peixes está indicada pela barra preta.

triturados em processador para homogeneização e a seguir liofilizados. Tendo em vista que a predação sobre esses peixes se dá partir da ingestão dos organismos inteiros, não foi retirada nenhuma porção da sua estrutura corporal para as análises. As análises bromatológicas foram conduzidas após o término do período amostral, quando todas as amostras estavam liofilizadas.

$\mathrm{O}$ valor de proteína bruta foi obtido pelo método de Kjeldahl (CUNNIFF, 1998) e o conteúdo protéico calculado pelo valor de nitrogênio de Kjeldahl x 6,25 . Os lipídios foram extraídos pelo método de Folch et al. (1957), e seu conteúdo determinado gravimetricamente. O teor de matéria mineral foi determinado gravimetricamente por incineração em mufla a $600^{\circ} \mathrm{C}$. O percentual de carboidrato foi estimado através da relação: $\mathrm{C}=100-(\% \mathrm{P}+\% \mathrm{~L}+\% \mathrm{M})$, onde $\mathrm{C}$ é carboidrato, $\mathrm{P}$ proteína, L lipídeo e $\mathrm{M}$ matéria mineral. Os valores da composição centesimal, expressos em porcentagem de massa seca, são médias de análises em triplicata com desvios padrões inferiores a $5 \%$. O valor de energia bruta (EB), expresso como kcal.g-1 de massa seca, foi determinado indiretamente através dos coeficientes de Rubner que consideram 5,65kcal.g ${ }^{-1}$ para proteína, 4,10kcal.g ${ }^{-1}$ para carboidrato e 9,45kcal.g ${ }^{-1}$ para lipídio (WINBERG, 1971), através da relação: EB $=(5,65 * \mathrm{P}+4,10 * \mathrm{C}+9,45 * \mathrm{~L}) / 100$.

$\mathrm{O}$ Teste $\mathrm{T}$ foi aplicado para verificar a diferença entre os períodos de amostragem (primavera-verão e outono-inverno) em relação os percentuais de proteína, lipídeo, matéria mineral, carboidrato e os valores de energia bruta, considerando todas as espécies estudadas. A Análise de Variância (ANOVA), seguida do teste de Tukey a posteriori, foi empregada para avaliar as diferenças entre essas variáveis, considerando as três espécies de Clupeiformes separadamente. A correlação de Pearson (r) foi utilizada para testar as correlações entre as variáveis e reuniu todas as espécies. As análises estatísticas foram realizadas no programa Statistica 5.5 for Windows e o valor de $p$ igual ou menor a 0,05 foi aplicado para indicar a significância das diferenças. 


\section{Resultados}

Os resultados da composição centesimal indicaram que a proteína é a maior fração da matéria orgânica em todas as espécies de Clupeiformes, seguida de carboidrato e lipídio (Tabela 1). A espécie L. grossidens foi representada pelos peixes de maior porte na malha amostral, que apresentaram percentual médio de lipídio cerca de duas e cinco vezes superior a $P$. harroweri e $C$. bleekerianus, respectivamente.

O baixo número amostral de $P$. harroweri e $L$. grossidens obtido no período primavera-verão e de $C$. bleekerianus obtido no outono-inverno não permitiu comparação sazonal intraespecífica em relação à composição centesimal e ao valor calórico (Tabela 1). No entanto, a comparação sazonal reunindo todas as espécies estudadas revelou valores significativamente maiores na primavera-verão para os percentuais de proteína $(\mathrm{t}=4,1575, p=0,00009)$ e energia bruta $(\mathrm{t}=$ $2,1762, p=0,03330)$ quando comparado ao período de outono-inverno.

As espécies analisadas foram significativamente distintas em relação ao porte, composição centesimal (com exceção do carboidrato) e energia bruta (Tabela 2). O percentual de proteína foi diretamente proporcional ao valor de energia bruta, enquanto o percentual de lipídio apresentou relação inversa com essas duas variáveis. As análises de correlação entre a composição centesimal e a energia bruta consideraram todos os peixes da amostragem e confirmaram os padrões registrados pela ANOVA (Tabelas 2 e 3 ).

TABELA 1: Caracterização das espécies de Clupeiformes quanto ao porte (comprimento total e peso), composição centesimal (proteína, lipídio, matéria mineral e carboidrato) e energia bruta (mín-máx; média \pm desvio padrão).

\begin{tabular}{lccc} 
& C. bleekerianus & P. harroweri & L. grossidens \\
\hline $\mathrm{N}^{\circ}$ Amostral & 20 & 3 & 6 \\
Primavera-verão & 3 & 19 & 14 \\
Outono-inverno & $9,9-11,3 ; 10,5 \pm 0,4$ & $9,8-13,8 ; 11,1 \pm 1,1$ & $9,3-14,4 ; 11,9 \pm 1,4$ \\
Comprimento (cm) & $5,2-8,3 ; 6,7 \pm 0,8$ & $7,6-26,8 ; 13,6 \pm 5,4$ & $12,2-24,4 ; 17,5 \pm 3,2$ \\
Peso (g) & $59,4-71,7 ; 66,1 \pm 2,7$ & $49,1-69,1 ; 58,4 \pm 4,1$ & $31,5-56,6 ; 50,3 \pm 6,6$ \\
Proteína (\% ms) & $1,5-10,4 ; 4,6 \pm 2,5$ & $2,8-25,0 ; 13,0 \pm 6,1$ & $10,3-42,8 ; 23,7 \pm 9,3$ \\
Lipídeo (\% ms) & $11,7-19,9 ; 15,0 \pm 1,8$ & $4,1-17,4 ; 13,5 \pm 2,9$ & $4,3-12,5 ; 7,8 \pm 2,4$ \\
Mineral (\% ms) & $8,5-19,6 ; 14,3 \pm 3,5$ & $4,3-23,7 ; 15,3 \pm 5,5$ & $5,2-35,8 ; 18,6 \pm 9,6$ \\
Carboidrato (\% ms) & $408,1-451,4 ; 432,3 \pm 11,4$ & $328,5-430,9 ; 395,5 \pm 27,4$ & $270,1-414,8 ; 362,8 \pm 41,8$ \\
EB (kcal.g- $\left.{ }^{-1} \mathrm{~ms}\right)$ & & &
\end{tabular}

EB: energia bruta; ms: massa seca.

TABELA 2: Análise de variância (ANOVA) do porte (comprimento total e peso), da composição centesimal (proteína, lipídio, matéria mineral e carboidrato) e da energia bruta das espécies de Clupeiformes.

\begin{tabular}{lccc} 
& $F$ & $p$ & Relação entre as espécies \\
\hline Comprimento (cm) & 9,243 & 0,000307 & C. bleekerianus; P. harroweri $<$ L. grossidens \\
Peso (g) & 48,282 & $<0,000001$ & C. bleekerianus $<$ P. harroweri $<$ L. grossidens \\
Proteína (\% ms) & 61,862 & $<0,000001$ & C. bleekerianus $>$ P. harroweri $>$ L. grossidens \\
Lipídeo (\% ms) & 46,802 & $<0,000001$ & C. bleekerianus $<$ P. harroweri $<$ L. grossidens \\
Mineral (\% ms) & 52,778 & $<0,000001$ & C. bleekerianus; P. harroweri $>$ L. grossidens \\
Carboidrato (\% ms) & 2,495 & 0,90673 & C. bleekerianus; P. harroweri; L. grossidens \\
EB (kcal.g $\left.{ }^{-1} \mathrm{~ms}\right)$ & 31,039 & $<0,000001$ & C. bleekerianus $>$ P. harroweri $>$ L. grossidens \\
\hline
\end{tabular}

EB: energia bruta; ms: massa seca. 
TABELA 3: Matriz de correlação entre os valores de composição centesimal (proteína, lipídio, matéria mineral e carboidrato) e de energia bruta.

\begin{tabular}{lcccc}
\hline & $\begin{array}{c}\text { Proteína } \\
(\% \mathrm{~ms})\end{array}$ & $\begin{array}{c}\text { Lipídeo } \\
(\% \mathrm{~ms})\end{array}$ & $\begin{array}{c}\text { Mineral } \\
(\% \mathrm{~ms})\end{array}$ & $\begin{array}{c}\text { Carboidrato } \\
(\% \mathrm{~ms})\end{array}$ \\
\hline $\begin{array}{l}\text { Lipídeo }(\% \mathrm{~ms}) \\
\text { Mineral }(\% \mathrm{~ms})\end{array}$ & $-0,691^{*}$ & & & \\
$\begin{array}{l}\text { Carboidrato } \\
(\% \mathrm{~ms})\end{array}$ & $-0,431^{*}$ & $-0,616^{*}$ & & \\
$\mathrm{~EB}\left(\mathrm{kcal.g} \mathrm{g}^{-1} \mathrm{~ms}\right)$ & $0,797^{*}$ & $-0,915^{*}$ & $-308^{*}$ & \\
\hline
\end{tabular}

$* p<0,05$; EB: energia bruta; ms: massa seca.

\section{Discussão}

Os Clupeiformes estudados pertencem à mesma guilda trófica, com dieta composta por crustáceos e peixes, que incluem componentes do zôoplancton e formas juvenis. A espécie C. bleekerianus se alimenta principalmente de crustáceos copépodes e sergestídeos, e as larvas e juvenis de peixes (Engraulidae e Clupeidae) também fazem parte da sua alimentação (SAZIMA et al., 2004; CORRÊA et al., 2005; MUTO et al., 2008). Devido à presença de dentes bem desenvolvidos, essa espécie pode se alimentar de peixes juvenis que alcançam até $50 \%$ do seu tamanho corporal (SAZIMA et al., 2004). Em relação a $P$. harroweri, Criales-Hernadéz (2003) e Muto et al. (2008) relatam a presença de crustáceos copépodes, ostracodes e decápodes como principais itens da dieta. A espécie L. grossidens é predominantemente piscívora, se alimentando de larvas e juvenis, mas crustáceos também fazem parte da sua alimentação (LOPES, 1998; BORTOLUZZI et al., 2006). Ao compartilharem a mesma área de uso na costa norte do Rio de Janeiro, espera-se que esses peixes tenham iguais possibilidades de acesso às fontes alimentares. No entanto, a comparação entre essas espécies quanto a composição centesimal e energia bruta foi significativamente distinta. Isso pode refletir o uso diferenciado dos recursos alimentares disponíveis, o que minimiza a competição interespecífica e a sobreposição de nicho alimentar.

Diferenças metabólicas específicas, tais como demanda nutricional e/ou energética e digestibilidade, também poderiam explicar os resultados obtidos pelo presente estudo. Eder e Lewis (2005), em estudo realizado com 27 espécies de teleósteos demersais e pelágicos que se distribuem no Oceano Atlântico Sul $\left(39^{\circ} \mathrm{S}-51^{\circ} \mathrm{S}\right)$, concluíram que a composição centesimal é fortemente relacionada a características específicas dos peixes. Adicionalmente, a composição de organismos marinhos pode variar com a localização geográfica, sazonalidade, porte, maturidade, sexo e regime alimentar (SAADETTIN et al., 1998; LAWSON et al., 1998). Nesse sentido, a condição (sexo, estado reprodutivo e grau de repleção estomacal) dos espécimes analisados no presente estudo também poderia ter influenciado nos padrões observados, mas não foram obtidos dados para a confirmação dessa inferência.

As proteínas são os principais constituintes da matéria orgânica presente no tecido dos peixes, podendo alcançar até $75 \%$ do seu peso seco. De modo geral, os peixes utilizam as proteínas para o crescimento, mas caso os níveis de lipídios não sejam suficientes para suprir a demanda energética, as proteínas também podem ser utilizadas como fonte de energia (WILSON, 2002). No presente trabalho, as fortes correlações entre proteína e valor calórico (positiva) e lipídio e valor calórico (negativa) indicam que as espécies estão suprindo a demanda energética preferencialmente através do conteúdo protéico. A comparação sazonal da composição centesimal e do valor calórico desses Clupeiformes indicou que tanto a proteína quanto a energia bruta registraram seus maiores valores no período de primavera-verão, reforçando a relação entre essas variáveis nos peixes estudados.

O principal papel dos lipídios, e especificamente dos ácidos graxos, é gerar energia metabólica na forma de adenosina trifosfato (ATP), sendo esses nutrientes a principal fonte de energia para a reprodução dos peixes (SARGENT et al., 2002). Heinsbroek et al. (2007), em estudo sobre metabolismo alimentar e composição do corpo da enguia européia, Anguilla anguilla, verificaram que os níveis de proteína diminuíram a medida em que houve aumento do conteúdo de lipídio, e que esse último aumentou proporcionalmente ao incremento do peso corporal dos peixes. A mesma relação foi constatada no presente estudo, onde os valores percentuais de proteína e lipídio foram inversamente proporcionais e 
L. grossidens, com conteúdo lipídico significativamente maior em relação aos demais peixes, apresentou comprimento e peso corporal superiores.

A espécie L. grossidens é anádroma, com movimentos migratórios relacionados à reprodução que ocorre em sistemas fluviais (FROESE; PAULY, 2010). Jørgensen et al. (1997), ao avaliarem o conteúdo lipídico do salmonídeo Salvelinus alpinus, concluíram que um pré-requisito para o sucesso da estratégia anádroma é a habilidade do peixe em utilizar condições favoráveis no ambiente marinho para o rápido crescimento e aumento da reserva de energia. Antes do período de migração para a desova, os autores verificaram o incremento médio do conteúdo lipídico da ordem de cinco vezes em machos e fêmeas. Estratégia semelhante foi registrada em outras espécies anádromas, como a truta arco-íris, Salmo gairdneri (JEZIERSKA et al., 1982) e a sardinha americana, Alosa sapidissima (LEONARD; MCCORMICK, 1999), e naquelas que realizam migrações reprodutivas dentro do mesmo ambiente, como o arenque, Clupea harengus (SLOTTE, 1999) e o atum, Thunnus thynnus thynnus (MOURENTE et al., 2002). Caula et al. (2008) compararam a composição centesimal e o valor calórico de peixes marinhos e de água doce comercializados no estado do Ceará, nordeste do Brasil, concluindo que os teores de gordura e os valores energéticos são maiores em peixes de água doce. É provável que a proporção mais elevada de lipídios em L. grossidens quando comparada as demais espécies estudadas seja reflexo na sua condição anádroma, tendo em vista que $P$. harroweri e C. bleekerianus se distribuem em águas marinhas e estuários onde passam todo o ciclo de vida (WHITEHEAD, 1985; FROESE; PAULY, 2010).

Aproximadamente $90 \%$ da matéria mineral presente nos peixes é constituída de cálcio e fosfato. O cálcio é fundamental na contração muscular e na constituição do esqueleto, enquanto o fosfato está relacionado ao equilíbrio osmótico e a formação e integridade óssea (LALL, 2002). Neste trabalho as amostras foram compostas por peixes inteiros, sem a retirada de vértebras, espinhas e trato digestivo e, dessa forma, podemos levantar a possibilidade de que a diferença de L. grossidens em relação às demais espécies se relaciona à densidade óssea do esqueleto e/ou ao grau de repleção estomacal dos espécimes analisados.

A proporção de carboidratos foi o único aspecto comum entre as três espécies estudadas. Em peixes, os carboidratos são armazenados na forma de glicogênio, mas essa não é uma fonte eficiente para obtenção de energia (ANTHONY et al., 2000). Isso foi confirmado, pois não houve correlação significativa entre o teor de carboidrato e o valor calórico das espécies.

A determinação dos valores energéticos das presas é importante na elaboração de modelos de transferência de energia entre níveis tróficos e na compreensão de mudanças das respostas tróficas dos predadores como consequência de mudanças na disponibilidade de alimento (BENOIT-BIRD, 2004; EDER; LEWIS, 2005). Dessa forma, os dados do presente trabalho servirão de base para estudos sobre bioenergética e para a interpretação da seletividade alimentar por parte de carnívoros marinhos que tem essas espécies de Clupeiformes incluídas em seu conjunto de presas preferenciais

\section{Agradecimentos}

Aos pescadores do porto de Atafona e à técnica de campo Silvana Ribeiro Gomes pelo auxílio na coleta dos peixes. Ao IBAMA pela concessão da licença de coleta permanente de material zoológico n 16401-1. D.R. Awabdi agradece ao CNPq pela concessão de bolsa de Iniciação Científica. A.P.M. Di Beneditto agradece à FAPERJ (E-26/103.038/08) e ao CNPq (30241/09-7 e 470002/10-7) pela concessão de bolsa de pesquisa e auxílio financeiro.

\section{Referências}

ACEVEDO-GUTIERREZ, A.; CROLL, D. A.; TERSHY, B. R. High feeding costs limit dive time in the largest whales. The Journal of Experimental Biology, Cambridge, v. 205, p. $1747-$ $1753,2002$.

ANTHONY, J. A.; ROBY, D. D.; TURCO, K. R. Lipid content and energy density of forage fishes from the northern Gulf of Alaska. Journal of Experimental Marine Biology and Ecology, Amsterdam, v. 248, p. 53-78, 2000.

BEARZIA, G.; POLITIA, E.; AGAZZIA, S.; AZZELLINO, A. Prey depletion caused by overfishing and the decline of 
marine megafauna in eastern Ionian Sea coastal waters (central Mediterranean). Biological Conservation, Hoboken, v. 127, p. 373-382, 2006.

BENOIT-BIRD, K. J. Prey caloric value and predator energy needs: foraging predictions for wild spinner dolphins. Marine Biology, Kiel, v.145, p. 435-444, 2004.

BITTAR, V. T.; CASTEllo, B. F. L.; DI BenEDitTO, A. P. M Hábito alimentar do peixe - espada adulto, Trichiurus lepturus, na costa norte do Rio de Janeiro, sudeste do Brasil. Biotemas, Florianópolis, v. 21, n. 2, p. 83-90, 2008.

BORTOLUZZI, T.; ASCHENBRENNER, A. C.; SILVEIRA, C. R.; ROOS, D. C.; LEPKOSKI, E. D.; MARTINS, J. A.; GOULART, M G.; QUEROL, E.; QUEROL, M. V. Hábito alimentar da sardinha prata, Lycengraulis grossidens (Spix \& Agassiz, 1829) (Pisces, Engraulidae), rio Uruguai médio, sudoeste do Rio Grande do Sul, Brasil. Biodiversidade Pampeana, Uruguaiana, v. 4, p. 11-23, 2006. BURKE, B. J.; RICE, J. A. A linked foraging and bioenergetics model for southern flounder. Transactions of the American Fisheries Society, Auburn, v. 131, p. 120-131, 2002.

CAMPO, D.; MOSTARDA, E.; CASTRIOTA, L.; SCARABELLO, M. P.; ANDALORO, F. Feeding habits of the Atlantic bonito, Sarda sarda (Bloch, 1793) in the southern Tyrrhenian sea. Fisheries Research, St John's, v. 81, p. 169-175, 2006.

CAULA, F. C. B.; OLIVEIRA, M. P.; MAIA, E. L. Teor de colesterol e composição centesimal de algumas espécies de peixes do estado do Ceará. Ciência e Tecnologia de Alimentos, Campinas, v. 28, p. 959-963, 2008.

CORRÊA, C. E.; CHAVES, P. T.; GUIMARÃES, P. R. B. Biology of Chirocentrodon bleekerianus (Poey,1867) (Clupeiformes: Pristigasteridae) in a continental shelf region of southern Brazil. Brazilian Archives of Biology and Technology, Curitiba, v. 48, p. 419-427, 2005.

CRIALES-HERNANDEZ, M. I. Diet composition of Pellona harroweri (Fowler) (Pisces: Pristigasteridae) in the Guajira Peninsula, Colombian Caribbean. Boletin de Investigaciones Marinas Costeras, Santa Marta, v. 32, p. 279-282, 2003.

CUNNIFF, P. A. Official methods of analysis of AOAC International. Arlington: Association of Official Analytical Chemists, 1998. CD-ROM.

CYTERSKI, M.; NEY, J.; DUVAL, M. Predator demand for clupeid prey in Smith Mountain Lake, Virginia. Fisheries Research, St John's, v. 59, p. 1-16, 2002.

DÄNHARDT, A.; BECKER, P. H. Does small-scale vertical distribution of juvenile schooling fish affect prey availability to surface-feeding seabirds in the Wadden Sea? Journal of Sea Research, Den Burg, doi:10.1016/j.seares.2010.11.002, 2011.

DENNERLINE, D. E.; VAN DEN AVYLE, M. J. Sizes of prey consumed by two pelagic predators in US reservoirs: implications for quantifying biomass of available prey. Fisheries Research, St John's, v. 45, p. 147-154, 2000.

DI BENEDiTTO, A. P. M.; RAMOS, R. M. A. Biology and conservation of the franciscana (Pontoporia blainvillei) in the north of Rio de Janeiro, Brazil. Journal of Cetacean Research and Management, Cambridge, v. 2, p. 185-192, 2001.

DI BENEDITTO, A. P. M.; SANTOS, M. V. B.; VIDAL JR., M. V. Comparison between the diet of two dolphins from south-eastern Brazil: proximate-composition and caloric value of prey species.
Journal of the Marine Biological Association of the United Kingdom, Plymouth, v. 89, p. 903-905, 2009.

DOYLE, T. K.; HOUGHTON, J. D. R.; MCDEVITT, R.; DAVENPORT, J.; HAYS, G. C. The energy density of jellyfish: estimative from bomb calorimetric and proximate-composition. Journal of Experimental Marine Biology and Ecology, Amsterdam, v. 343, p. 239-252, 2007.

EDER, E. B.; LEWIS, M. N. Proximate composition and energetic value of demersal and pelagic prey species from the South-West Atlantic Ocean. Marine Ecology Progress Series, Oldendorf, v. 291, p. 43-52, 2005.

EGGLETON, M. A.; SCHRAMM JR., H. Feeding ecology and energetic relationships with habitat of blue cattfich, Ictalurus furcatus, and flathead catfish, Pylodictis olivaris, in the lower Mississippi River, U.S.A. Environmental Biology of Fishes, Dordrecht, v. 70, p. 107-121, 2004.

FAO. FAO yearbook: Fishery and Aquaculture Statistics 2008. Rome: Food and Agriculture Organization, 2010. 72 p.

FIGUEIREDO, J. L.; MENEZES, N. A. Manual de peixes marinhos do Sudeste do Brasil. II. Teleostei (1). São Paulo: Museu de Zoologia da Universidade de São Paulo, 1978. 110 p.

FOLCH, J.; LEES, M.; STANLEY, S. A simple method for the isolation and purification of total lipids from animal tissues. Journal of Biological Chemistry, La Jolla, v. 226, p. 497-509, 1957.

FROESE, R.; PAULY, D. Fish Base. Word Wide Web electronic publication. 2010. Disponível em: <www.fishbase.org >, version (11/2010). Acesso em: 24 abr. 2010.

HEINSBROEK, L. T. N.; VAN HOOFF, P. L.A.; SWINKELS, W.; TANCK, M. W. T.; SCHRAMA, J. W.; VERRETH, J. A. J. Effects of feed composition on life history developments in feed intake, metabolism, growth and body composition of European eel, Anguilla Anguilla. Aquaculture, Amsterdam, v. 267, p. $175-$ $187,2007$.

JEZIERSKA, B.; HAZEL, J. R.; GERKING, S. D. Lipid mobilization during starvation in the rainbow trout, Salmo gairdneri Richardson, with attention to fatty acids. Journal of Fish Biology, Dumfries, v. 21, p. 681-692, 1982.

JØRGENSEN, E. H.; JOHANSEN, S. J. S.; JOBLING, M. Seasonal patterns of growth, lipid deposition and lipid depletion in anadromous Arctic charr. Journal of Fish Biology, Dumfries, v. 51, p. 312-326, 1997.

LALL, S. P. The minerals. In: HALVER, T. E.; HARDY, R. W. (Eds). Fish Nutrition. 3 ed. San Diego: Academic Press, 2002. p. 259-308.

LAWSON, J. W.; MAGALHÃES, A. M.; MILLER, E. H. Important prey species of marine vertebrate predators in the northwest Atlantic: proximate composition and energy density. Marine Ecology Progress Series, Oldendorf, v. 164, p. 3-20, 1998.

LEONARD, J. B. K.; MCCORMICK, S. D. Effects of migration distance on whole-body and tissue-specific energy use in American shad (Alosa sapidissima). Canadian Journal of Fisheries and Aquatic Sciences, Toronto, v. 56, p. 1159-1171, 1999.

LOPES, P. R. D. Nota sobre alimentação de Lycengraulis grossidens (Agassiz, 1829) (Osteichthyes, Clupeiformes, Engraulidae) na Praia de Jaguaribe (Ilha de Itamaracá) Pernambuco. Acta Biologica Leopoldensia, São Leopoldo, v. 20, n. 2, p. 243-250, 1988. 
MOURENTE, G.; MEGINA, C.; DÍAZ-SALVAGO, E. Lipids in female northern bluefin tuna (Thunnus thynnus thynnus L.) during sexual maturation. Fish Physiology and Biochemistry, Amsterdam, v. 24, p. 351-363, 2002.

MUTO, E. Y.; MALFARA, D. T.; COELHO, L. I.; SOARES, L.

S. H. Alimentação das sardinhas Pellona harroweri (Fowler, 1919) e Chirocentrodon bleekerianus (Poey, 1867), na região costeira de Santos, Estado de São Paulo. In: BRAGA, E. S. (Ed.). Oceanografia e mudanças globais. 1 ed. São Paulo: Instituto Oceanográfico da Universidade de São Paulo, 2008. p. 287-302.

NILSSEN, K. T. Seasonal distribution, condition and feeding habits of Barents Sea harp seals (Phoca groenlandica). In: BLIX, A. S.; WALLØE, L.; ULLTANG, Ø (Eds). Whales, seals, fish and man. Amsterdam: Elsevier Science, 1995. p. 241-254.

SAADETTIN, G.; DINCER, B.; ALEMDAG, N.; COLAK, A.; TUFEKCI, M. Proximate composition an selected mineral content of commercially important fish species from the Black Sea. Journal of the Science of Food and Agriculture, Davis, v. 78, p. 337-342, 1998.

SARGENT, J. R.; TOCHER, D. R.; BELL, J. G. The lipids. In: HALVER, T. E; HARDY, R. W. (Eds). Fish Nutrition. 3 ed. San Diego: Academic Press, 2002. p.181-257.
SAZIMA, C.; MOURA, R. L.; SAZIMA, I. Chirocentrodon bleekerianus (Teleostei: Clupeiformes: Pristigasteridae), a small predaceous herring with folded and distinctively oriented prey in stomach. Brazilian Journal of Biology, São Paulo, v. 64, n. 1, p. 165-168, 2004.

SLOTTE, A. Differential utilization of energy during wintering and spawning migration in Norwegian spring-spawning herring. Journal of Fish Biology, Dumfries, v. 54, p. 338-355, 1999.

WHITEHEAD, P. J. P. FAO species catalogue. v. 7. Clupeoid fishes of the world. An annotated and illustrated catalogue of the herrings, sardines, pilchards, sprats, anchovies, and wolf herrings. Part I. Chirocentridae, Clupeidae and Pristigasteridae. Rome: Food and Agriculture Organization, 1985. 303 p.

WILSON, R. P. Amino acids and proteins. In: HALVER, T. E; HARDY, R. W. (Ed.). Fish Nutrition. 3 ed. San Diego: Academic Press, 2002. p. 143-179.

WINBERG, G. G. Methods for estimation of production of aquatic animals. London: Academic Press, 1971. 175 p. 